\title{
Organizational Sustainability and Value Creation in Collaborative Networks
}

\author{
Pierluca Di Cagno, Bianca D’Agostinis, and Barbara Livieri \\ Department of Economic Sciences, \\ University of Salento, Lecce, Italy \\ \{name.surname\}@unisalento.it
}

\begin{abstract}
Collaborative Networks are becoming ever more important as a lunchpad for the achievement of competitive advantages and for the creation of socio-economic benefits. According to the relational view theory, joint efforts can indeed generate relational rents. However, task coordination is not a sufficient means for value creation. Indeed, Collaborative Networks have to create a link between several aspects, such as trust, culture of collaboration, knowledge sharing, managerial processes, incentive systems, ethical code and so on in order to create cooperation and, thus, value. Aim of this work is to analyze how these aspects affect each other and how they affect value creation within collaborative networks. In order to do so, we develop a model, based on UML and e3value, in which the main factors impacting on value creation and value exchanges within CNOs are represented. After the description of the model, we analyze a case study of a CNO.
\end{abstract}

Keywords: Collaborative Networks, Value Creation, Sustainability, e-3 value, Value exchanges, Relational rents.

\section{Introduction}

In the actual economic context, Collaborative Networks (CNOs) represents a lunchpad for the enhancement of firms' competitiveness and for the creation of socioeconomic and environmental benefits. CNOs can be seen as a system made by several "entities that are largely autonomous, geographically distributed, and heterogeneous in terms of their operating environment, culture, social capital and goals [...]", enabled by new technologies [1]. In this scenario, nodes of a CNO share information, resources and competencies with the aim of create new value through the broadening of enterprise boundaries.

However, the sustainability of CNOs, from not only a financial perspective but also from the organizational one is the premise for value creation. Indeed, collaboration implies mutual trust and commitment, giving relevance to behavioral and managerial principles, ethic codes, collaboration culture and incentive systems. Several authors have studied the role of trust and commitment in inter-organizational settings [2, 3], however, at the best of our knowledge, no one of them studied these factors with a systematic approach that led to modeling and requirements. Nonetheless, CNOs are 
complex entities, whose understanding is not immediate, therefore it becomes necessary to understand and model the determinants of value creation in CNOs [4].

In this work, we propose a model of sustainability and value creation within CNO. In particular, we represent these aspects by means of UML and e3-value, an ontology based methodology used to define requirements for software systems taking into account the value exchange among different individuals. In this way, we were able to model and analyze value exchanges and co-creation in Collaborative Networks.

The works is structured as follows. Section 2 is for the analysis of related works concerning the use of e3value. In Section 3 we pose the basis for our model, which we present, by means of a visual representation, in Section 4. In Section 5 we propose a case study. Finally, in Section 6 we discuss our model and the application to the case study and we draw conclusions.

\section{Related Works}

The advent of e-business has led to the necessity of the development of e-business models. The e-business model has to address the formulation of requirements through a rigorous method [5]. In this scenario, Gordijn and Akkermans [5, 6] proposed a multi-viewpoint approach for the business model development: a) value viewpoint, that represents the value creation and exchange process; b) process viewpoint, that represents business process and tasks; c) Information System viewpoint, that describes the Information Systems required for business processes.

From a value point of view, e3-value is commonly used for the modeling of value exchanges. The e3-Value is in fact an ontology-based methodology for defining business models for business networks [5] incorporating concepts from requirements engineering and conceptual modeling (including a graphical notation). Its main focus is on identifying and analyzing how value is created, exchanged and consumed within a multi-actor network. Hence, taking the economic value perspective and visualizing what is exchanged, i.e. which kind of economic value, and by whom [7]. An economic value exchange, and consequently the e3-value ontology as a whole, is based on the principle of reciprocity emphasizing the dual character of business transactions. This "give and take"- approach, described by means of specific "value-exchange diagrams", denotes that every actor offers something of value, such as money, physical goods, services, or capabilities, and gets a value in return. In this scenario moneyexchange aspects are included in the value-exchange diagrams, and this semi-formal description is very useful for business analysis [5] but also for requirement specification on ePayments and for other aspects (decision making, user profiling, market segmentation, ...) directly connected to monetization in eServices. This approach can bring several benefits, such as better communication among actors, improvement of the decision making process and a more complete view of business operations and business perspective. In particular, when applied to CNOs can help in the understanding of value exchanges among partners and external actors, thus extending existing reference models, such as ARCON. 


\section{A Collaboration Model: Theoretical Foundation}

In literature, several types of partnerships among firms have been identified, characterized by the deepness of the sharing relations and by the integration level of firms. In particular, Camarinha-Matos and Afsarmanesh [1,8] identified the following integration levels: (a) networking, which consists of the information exchange for mutual benefits, with no common goals; (b) coordination, which consists of the coordination of autonomous tasks, connected to a common goal; (c) cooperation, which consists in resource and information sharing, with the aim of achieving compatible goals; (d) collaboration, which is the highest integration level and consists of the sharing of information, resources and responsibility in order to organize and develop activities for a common goal.

Only in this last sense we can talk of collaborative network which have been defined as "a network consisting of a variety of entities that are largely autonomous, geographically distributed, and heterogeneous in terms of their operating environment, culture, social capital and goals, but that collaborate to better achieve common or compatible goals, and whose interactions are supported by computer network". [1]. CNOs enable several benefits, among which the increase in the success rate of enterprises, also in turbulent markets, and the increased capability to achieve shared goals [1]. Indeed, through alliances, it is possible to enhance enterprise size, gain access to new markets and to external know-how, create synergies, share resources and risks, integrate firms' resources with the ones of partners and enhance innovation processes, with a result of increased value creation [9]. Collaboration among enterprises enable the finding of resources not easily accessible on the market or that cannot be easily created. Also, the interactions among partners can be generated the relational capital [10], defined as a set of relations among firms which facilitate the exploitation of economic activities. Resources are exchanged when this is mutually convenient and there is mutual trust, which exists when: a) partners confide in a reliable and transparent exchange of resources; b) a firm believes that other partners are pursuing actions that are beneficiary also for the firms itself and c) that these actions are not prejudicial [11]. Indeed, trust has been seen as "the cornerstone of strategic partnership" [12], whereas the lack of trust can bring to the failure of the alliance [13] and whereas if associated with commitment can bring to "outcomes that promote efficiency, productivity and effectiveness" [14].

In the field of strategic alliances, trust is often a cognition-based trust, which comes from rational choices based on information, rather than from emotional bonds between individuals (affect-based trust), that follow in case of recurring interactions between firms. In particular, cognition-based trust comes from previous interactions, reputation on the market, ecological and ethical responsibility, organizational context, similarities in firms' characteristics. The searching for trust based relations have to rationales: a) CNOs with a high level of trust are more likely to succeed and to create value; b) relations among partners with a high level of trust requires much less time and commitment to be managed [15]. For these reasons, the partner selection is often based on previous interactions, reputation and potential degree of conflict [15]. 
Recognizing the importance of model frameworks for CNO, the ARCON model was developed by $[4,16]$. In the model, four endogenous elements are analyzed, namely structural dimension, componential dimension, functional dimension, behavioral dimension. The behavioral dimension regards the principles, policies and rules of governance that guide who operated within the CNO in order to make it more efficient [17]. Indeed, CNO governance is particularly relevant, since it's a requirement for the effective monitoring and control of relations. Inter-organizational relations create a not-planned operative context, which at the same time is not entirely spontaneous [18]. Moreover, another relevant element is risk, which has been analyzed towards three perspectives. In [19] firms choose to collaborate in order to face market risks; in [17] risk is the result of CNOs, while in [20] trust derives from risk and, at the same time, is affected by trust. However, cooperation could lead to a reputational risk, coming from non-socially responsible behavior of partners that could affect the whole CNO.

In short, the advantages of CNOs are fully exploited only when there is trust among partners, an adequate governance, ethical codes and a culture of collaboration.

\section{SuMCNOs: A CNOs Sustainability Model}

\subsection{Method}

For the development of our model, we selected UML as means to represent general factors and variable, and e3-value for the analysis of value exchanges in CNOs, since it's a business ontology specifically designed for the analysis of value constellations [5]. After reviewing more than 50 papers related to CNOs, published on international conferences and journals, we identified three variables that have a great impact on value creation. We modeled these variables with a UML class diagram. To this purpose, we considered the nodes of the networks, the CNOs and the variables that impact on value creation as classes, since a class is an abstraction of things (or instances): in other words, classes represent a whole set of objects that share methods and attributes [21]. Moreover, we analyzed the value exchanges within networks by means of e3value. To this aim, we modeled CNOs as composite actors made by other business actors. Accordingly to e3value standard notation, we show the value activities that contribute to reaching the CNOs goals as value activities, while the value exchanges have been depicted as connection between value ports, assigned to each actor. Finally, we applied this model to a case of study, for which we draw the corresponding e-3 value diagram. All the diagrams are drawn with Microsoft Visio and e3value Editor.

\subsection{Model Representation}

This section provides a representation of our proposal, in order to exemplify our modeling approach. In the first part of the model, based on UML, we represent the determinants that impact on value creation and exchange within CNOs. As stated in the theoretical foundation section, the main aspects concern trust, resource sharing and 
commitment. For the purpose of this study, we intend value as the result of the activities of the individual firm and of the collaboration with other entities. In this sense, the value refers not only to financial benefits, but also to socio-environmental ones.

In Fig. 1, we represent the candidate classes and the candidate associations of our model. In particular, each Node is part of a CNO; both Node and CNO are children of the parent class BusinessEntity, which is a generalization of their concepts. Also, nodes share Resource, which is a generalization of TangibleResource, FinancialResource and IntangibleResource. To the primary association between Node and Resource, we tied an association class Sharing since the association is, in turn, associated with other classes. In a similar way, to the primary association between Node and CNO, we tied an association class Participating since the association itself has some attributes, namely Trust, Commitment and Relationalcapital. We choose to represent these attributes as classes, in order enable the association with other classes. In more detail, Trust is affected by several factors, whereas the Factor class is a generalization of InternalFactor, which is an attribute of the association class Participating, and Externalfactor. Relationalcapital, which is an attribute of Participating, is also associated with the class BusinessEntity. Tying RelationalCapital both to the class Participating and to the class BusinessEntity enables its attribution to the level on which the information is available.

Moreover, Relationalcapital is enabled by:

- Trust and Commitment, which are attributes of the class Participating: this relation doesn't need to be represented, since are all attributes of the same association class, therefore the association it's implicit;

- The Sharing of resources: if nodes don't share their resources, it's difficult to find relational capital, since it's strictly linked with how much firms work togheter.

The associate class Sharing, in turn, is enabled by Commitment and Trust, as explained in Section 3.

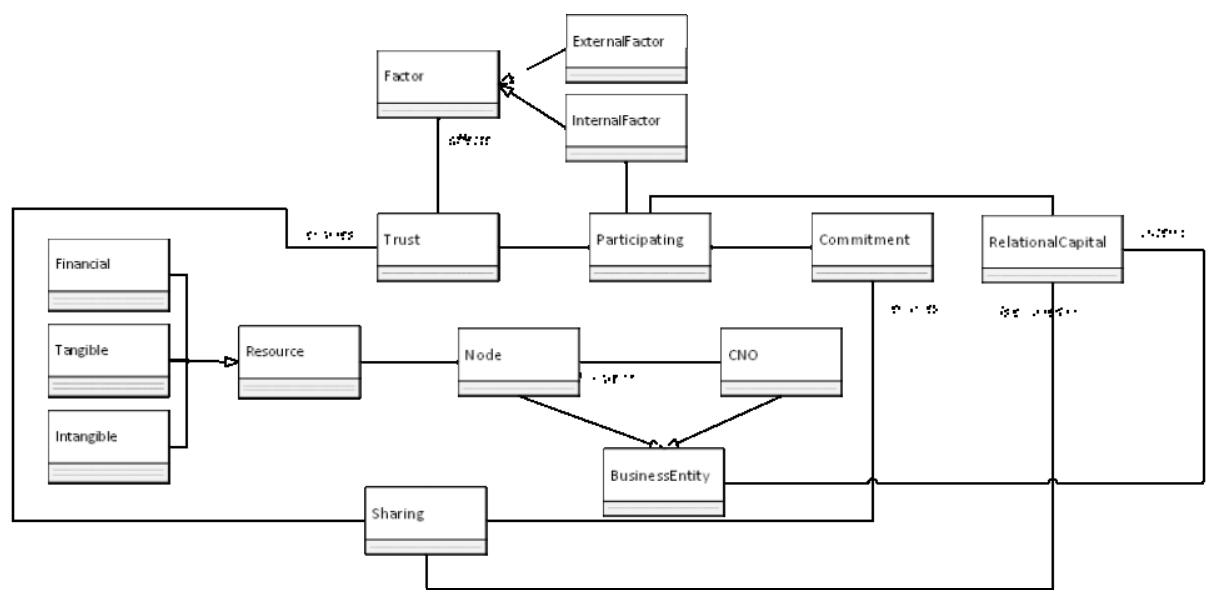

Fig. 1. UML class diagram representing value creation in CNOs 
The second part of the model is represented by means of e3 value ontology, through which we depict the value exchanges inside and outsides the collaborative network. To this purpose, we take into account two types of CNOs and, in particular, a supply chain and a horizontal strategic alliance.

In Fig. 2, value exchanges in a supply chain are represented. SupplyChain is a composite actor made by the actors Supplier and Internalcustomer. Supplier has a value activity Production and sell its products and services (value link) to Internalcustomer, who in exchange pay the price (money) to the supplier. Supplier also sells its product towards ExternalCustomer: the benefits of the $\mathrm{CNO}$ with respect to a market-driven environment is that products and services within the alliance can be sold at a lower price or can be more specific for the customer's needs. Also, Internalcustomer has a value activity Production and sell its final products and services to FinalCustomer. The social benefit is that CNO, under certain circumstances, enhance products quality and reduce prices.

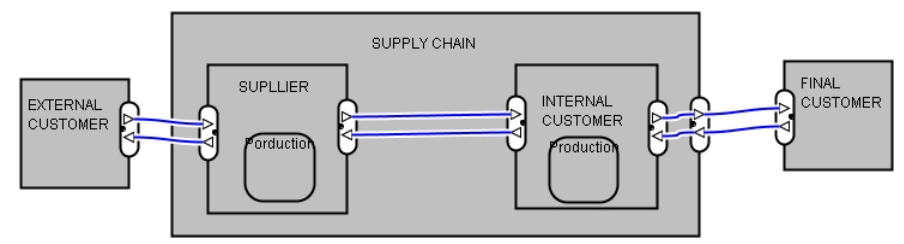

Fig. 2. Representation of value exchanges in CNOs

Moreover, exchanges within a horizontal alliance are represented. As depicted in Fig. 3, the value exchanges don't happen between the partners of the CNOs, but between the nodes and the CNO itself, whereas the benefits for nodes are usually intangibles.

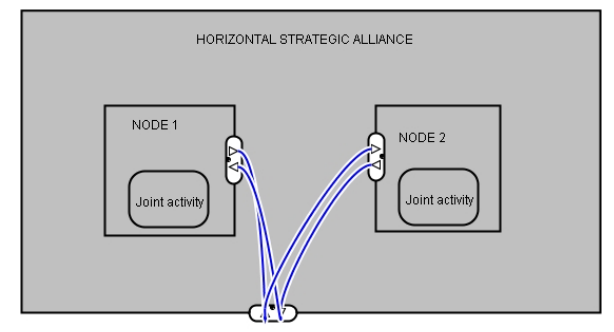

Fig. 3. Representation of value exchanges of horizontal strategic alliances

\section{$5 \quad$ Case Study}

The Network of Bathing Establishments of Viareggio is a collaborative network born in 2011 with the aim of increasing the competitiveness of the participants (85 bathing establishments). The main objectives of the network include (a) the increase of security on the beaches, (b) the improvement of environmental sustainability and (c) the coordination in supplying services. 
For the purpose of the analysis of value creation, we will analyze a specific project realized by the CNO regarding the safety of bathing establishments. The projects enabled services of medical aid and first support with AED (Automated External Defibrillator). In order to achieve this result, the network bought 41 defibrillators and offered courses for about 150 staff operators. As depicted in Fig. 3, Node1 and Node2 pay a fee in order to access to the project, whilst NetworkOfBathingEstablishments buys from AEDSupplier 41 defibrillators and from CouseErogator a service (course offering), under the payment of a fee. Finally, NetworkofBathingEstablishments offers this service and the defibrillators to Node1 and Node2. This enables Node1 and Node2to offer more services to their clients, which in return will get a safer environment also for elderlies and for people with health problems.

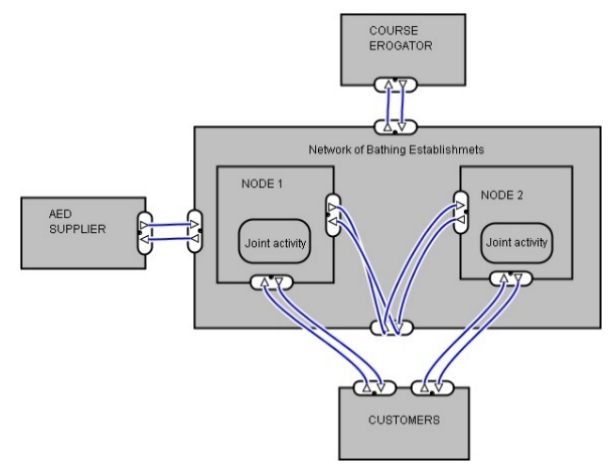

Fig. 4. Representation of the value exchanges in the Network of Bathing Establishments

In this case, trust mechanisms between nodes have been enabled by the continuous interaction among partners and by the network manager, who helped in the switch from a internal-based culture, where every external entity is a competitor, towards a collaborative culture, where each node is seen as relevant part for value creation for the $\mathrm{CNO}$ and for the creation of social benefits.

\section{Discussion and Conclusions}

In this paper, we propose a model for collaborative networks that takes into account both the factors affecting value creation and the value exchanges within the network. In particular, we described by means of UML the role of commitment and trust in the participation relationship and the variables that affect these two factors. Also, we analyzed through e3value the value exchanges within CNOs taking into account two different scenarios, namely a supply chain and a horizontal strategic alliance. Finally, we used e3value ontology in order to describe a case study of a horizontal network between bathing establishments. Whilst previous research only analyzed single enabling factors or value chains, we jointly examined these perspectives and we developed a model, named SuMCNOs, which can be easily applied to different cases in order to 
understand the sustainability of CNOs. Future works will include the extension of the scenarios investigated.

\section{References}

1. Camarinha-Matos, L.M., Afsarmanesh, H.: Collaborative networks: Value creation in a knowledge society. In: Wang, K., Kovacs, G., Wozny, M., Fang, M. (eds.) Knowledge Enterprise: Intelligent Strategies In Product Design, Manufacturing, and Management. IFIP, vol. 207, pp. 26-40. Springer, Boston (2006)

2. Essa, S.A.G., Dekker, H.C., Groot, T.: The Influence of Information and Control on Trust Building in Buyer-Supplier Negotiations (2013)

3. Bierly, P.E., Gallagher, S.: Explaining Alliance Partner Selection: Fit, Trust and Strategic Expediency. Long Range Plann. 40, 134-153 (2007)

4. Camarinha-Matos, L.M., Afsarmanesh, H.: A comprehensive modeling framework for collaborative networked organizations. J. Intell. Manuf. 18, 529-542 (2007)

5. Gordijn, J., Akkermans, H., Van Vliet, J.: Designing and evaluating e-business models. IEEE Intell. Syst. 16, 11-17 (2001)

6. Gordijn, J., Van Eck, P., Wieringa, R.: Requirements Engineering Techniques for eServices. Serv. Comput. Coop. Inf. Syst. Ser., 331-352 (2009)

7. Akkermans, J.M., Gordijn, J.: Value-based requirements engineering: exploring innovative e-commerce ideas. Requir. Eng. 8, 114-134 (2003)

8. FInES Task Force on Collaborative Networks and SOCOLNET: Taxonomy of Collaborative Networks Forms (2012)

9. Lavie, D.: The Competitive Advantage of Interconnected Firms: An Extension of the Resource-Based View. Acad. Manag. Rev. 31, 638-658 (2006)

10. Burt, R.S.: The network structure of social capital. Res. Organ. Behav. 22, 345-423 (2000)

11. Anderson, J.C., Narus, J.A.: A model of distributor firm and manufacturer firm working partnerships. J. Mark. 54 (1990)

12. Spekman, R.E.: Strategic supplier selection: understanding long-term buyer relationships. Bus. Horiz. 31, 75-81 (1988)

13. Sherman, S.: Are strategic alliances working? Fortune 126, 77-78 (1992)

14. Morgan, R.M., Hunt, S.D.: The commitment-trust theory of relationship marketing. J. Mark. 58 (1994)

15. Kwon, I.G., Suh, T.: Factors affecting the level of trust and commitment in supply chain relationships. J. Supply Chain Manag. 40, 4-14 (2004)

16. Camarinha-Matos, L.M., Afsarmanesh, H.: Collaborative Networks: Reference Modeling. Springer (2008)

17. McAllister, D.J.: Affect-and cognition-based trust as foundations for interpersonal cooperation in organizations. Acad. Manag. J. 38, 24-59 (1995)

18. Benassi, M.: Governance factors in a network process approach. Scand. J. Manag. 11, 269-281 (1995)

19. Boon, S.D., Holmes, J.G.: The dynamics of interpersonal trust: Resolving uncertainty in the face of risk. Coop. Prosocial Behav. 190-211 (1991)

20. Das, T.K., Teng, B.-S.: Trust, control, and risk in strategic alliances: An integrated framework. Organ. Stud. 22, 251-283 (2001)

21. Rumbaugh, J., Jacobson, I., Booch, G.: The Unified Modeling Language Reference Manual. Pearson Higher Education (2004) 